\title{
A simplified approach to haemoptysis
}

\author{
Zi Yang Trevor $\underline{\text { Ong }}{ }^{1,2}$, Hui Zhong $\underline{C h a i}^{2}$, MBBS, MRCP, Choon How $\underline{H o w}^{3}$, MMed, FCFP, Jansen $\underline{K o h}^{2}$, MRCP, FCCP,
}

Teck Boon Low ${ }^{2}$, MRCPI, FRCPI

Mrs Lim, a 55-year-old homemaker whom you last saw two years before, visited your clinic complaining of blood in her sputum when she clears her throat. She coughed up approximately $10 \mathrm{~mL}$ of bright red blood in the morning on the day of her visit. She had no complaints of chest pain or shortness of breath. Mrs Lim has a past medical history of hypertension and was diagnosed with type 2 diabetes mellitus during her recent hospital admission for acute appendicitis. She does not smoke and has no family history of lung disease.

\section{WHAT IS HAEMOPTYSIS?}

Haemoptysis is defined as the expectoration of blood originating from the tracheobronchial tree or pulmonary parenchyma. ${ }^{(1)}$ It can be classified as massive or non-massive based on the volume lost. Massive haemoptysis has been described as blood loss ranging from 100-600 $\mathrm{mL}$. However, for the purpose of this article, massive haemoptysis is defined as loss of at least $200 \mathrm{~mL}$ of blood in 24 hours or $50 \mathrm{~mL}$ per episode. ${ }^{(2,3)}$ Although massive haemoptysis accounts for only $5 \%$ of haemoptysis cases, it is an alarming symptom with an associated mortality of more than $50 \% .^{(4)}$

\section{HOW RELEVANT IS THIS TO MY PRACTICE?}

Haemoptysis is a common presentation in the primary, secondary and tertiary care setting. ${ }^{(5)}$ Hence, it is essential for primary care physicians to be well-versed in the evaluation and management of patients with haemoptysis. This article aimed to discuss the more common differential diagnosis, indications for referral to the emergency department or specialty clinics, and a general management plan for patients with non-massive haemoptysis.

\section{WHAT CAN I DO IN MY PRACTICE?}

In the primary care setting, it is important to differentiate between pseudohaemoptysis and haemoptysis. Thereafter, the patient's clinical status needs to be assessed, as well as the quantity of blood lost. The physician should then attempt to identify the cause of the bleeding and determine if a specialist or inpatient referral is needed.

\section{True haemoptysis versus pseudohaemoptysis}

When evaluating a patient presenting with expectoration of blood, one must determine the source of the bleeding and determine whether the patient is presenting with true haemoptysis or pseudohaemoptysis. Aetiologies of pseudohaemoptysis include the upper gastrointestinal tract and upper respiratory tract (Table I). . $^{(6,7)}$

\section{Differential diagnosis for haemoptysis}

There are multiple causes of haemoptysis. This article focuses on the more common causes such as (a) infective; (b) neoplastic; (c) vascular; (d) autoimmune; and (e) drug-related. A more detailed differential list can be found in Table II. It is also important to assess for any respiratory comorbidities while evaluating patients with haemoptysis.

\section{Infective causes}

Pneumonia is a common cause of haemoptysis; in such patients, physicians must look for symptoms and signs of infection such as fever, chest pain and productive cough. It is also important to obtain any relevant contact and/or travel history. Tuberculosis is a common cause of massive haemoptysis, with an incidence rate of 38 per 100,000 population in 2015. ${ }^{\left({ }^{(8)}\right.}$ It is hence prudent for a practitioner to look for risk factors of tuberculosis. This includes immunosuppression, which may be secondary to immunosuppressive medications or conditions such as human immunodeficiency virus infection or diabetes mellitus.

\section{Neoplastic causes}

Neoplastic lesions that can cause haemoptysis can be divided into primary pulmonary lesions and metastatic lesions. If a malignant cause is suspected, physicians should consider a thorough history and examination to look for other primary malignancies such as history of breast, kidney, gastrointestinal, ovarian and cervical cancers. ${ }^{(9)}$ It is important to also elicit constitutional symptoms such as loss of weight and appetite, and to consider the risk factors for primary lung carcinoma such as smoking and occupational exposure.

\section{Vascular causes}

Vascular causes of haemoptysis include pulmonary embolism. In patients with suspected pulmonary embolism, the physician should ask if they have a history of recent surgery or immobilisation. ${ }^{(10)}$ The limbs should be assessed for any signs of deep vein thrombosis as 
Table I. Differentiating features of pseudohaemoptysis.

\begin{tabular}{llll}
\hline Aetiology & History & Physical findings & Laboratory test \\
\hline Upper gastrointestinal tract & $\begin{array}{l}\text { Coffee ground vomitus, } \\
\text { black tarry stools, }\end{array}$ & $\begin{array}{l}\text { Epigastric tenderness, signs of } \\
\text { chronic liver disease (spider }\end{array}$ & $\begin{array}{l}\text { Acidic blood pH, blood mixed with } \\
\text { food, oesophago-gastro-duodenoscopy, } \\
\text { blood in nasogastric aspirate }\end{array}$ \\
& $\begin{array}{l}\text { naevi, palmar erythema) } \\
\text { Uppeeding gums, }\end{array}$ & $\begin{array}{l}\text { Gingivitis, telangiectasias, } \\
\text { phistaxis, no cough or }\end{array}$ & Nasopharyngoscopy \\
& sputum, sore throat & & \\
\hline
\end{tabular}

Table II. Differentials for haemoptysis.

\begin{tabular}{ll}
\hline Infective & Pneumonia \\
& Bronchiectasis \\
& Fungal infection \\
& Lung abscess \\
& Tuberculosis/nontuberculous mycobacteria \\
Neoplastic & Primary lung cancer \\
& Lung metastasis \\
& Bronchial adenoma \\
Vascular & Pulmonary embolism \\
& Pulmonary arteriovenous malformation \\
Autoimmune & Systemic lupus erythematosus \\
& Goodpasture syndrome \\
& Wegener's granulomatosis \\
& (granulomatosis with polyangiitis) \\
& Diffuse alveolar haemorrhage \\
& Anticoagulant \\
Drug-related & Antiplatelet agent \\
and others & latrogenic cause \\
& Lung contusion/penetrating injury \\
\hline
\end{tabular}

well. Other signs and symptoms to look out for include dyspnoea, chest pain, tachypnoea and tachycardia. ${ }^{(11)}$

\section{Autoimmune causes}

Vasculitic rash, haematuria, joint pain or swelling may be suggestive of underlying autoimmune diseases such as Wegener's granulomatosis (granulomatosis with polyangiitis), systemic lupus erythematosus (SLE) or Goodpasture syndrome. ${ }^{(12,13)}$ The characteristic butterfly rash or other skin lesions such as alopecia in SLE may also be found. ${ }^{(14)}$ If autoimmune disease is suspected, the patient should be referred to a specialist centre for further evaluation.

\section{Drug-related causes}

Common drugs that may cause haemoptysis include anticoagulants and antiplatelet agents. It is important to elicit further history (i.e. medication history) in order to predict the consequence of stopping medications as part of management.

\section{HOW SHOULD I APPROACH HAEMOPTYSIS?}

When approaching a patient with haemoptysis, a proper history has to be taken to narrow down the aetiology of the patient's symptoms. The physician should first exclude the possibility of pseudohaemoptysis and then narrow down the groups of causes for true haemoptysis. It is also paramount to quantify the amount of blood loss and evaluate for any complications due to blood loss. This includes evaluation of signs and symptoms of anaemia and searching for signs of haemodynamic compromise. The patient's vital signs should be measured and documented. Criteria for admission to the emergency department or referral to specialist clinics are as follows: ${ }^{(6,15)}$ (a) high risk of massive bleed; (b) gas exchange abnormalities (respiratory rate $>30$ breaths/minute, oxygen saturation $<88 \%$ room air); (c) haemodynamic instability (e.g. tachycardic, tachypnic, hypotensive patient); (d) other respiratory comorbidities (e.g. previous pneumonectomy, chronic obstructive pulmonary disease); and (e) other comorbidities (e.g. ischaemic heart disease, need for anticoagulants/antiplatelet agents).

Chest radiography is typically recommended for all patients who present with haemoptysis. It is a quick, readily available and cheap modality that can assist in revealing any focal or diffuse parenchymal involvement as well as pleural abnormalities. ${ }^{(4)}$ Other diagnostic modalities include bronchoscopy, multidetector computed tomography (CT), multidetector CT angiography and digital subtraction angiography.

\section{HOW SHOULD I MANAGE HAEMOPTYSIS?}

When a patient with non-massive haemoptysis has no haemodynamic instability, a normal chest radiograph and low risk of massive bleeding in the acute setting, no additional testing is required. In a patient with increased risk of malignancy (i.e. 30 pack-years of smoking, age $\geq 40$ years), a referral for CT should be considered (Fig. 1). ${ }^{(6)}$ In a case of massive haemoptysis with haemodynamic instability, the patient's airway, breathing and circulation should be managed first. Patients should be placed in the lateral decubitus position with the affected lung in a dependent position to avoid pooling of blood in the unaffected lung. They should be referred to hospital immediately for further management, including immediate airway control with rigid bronchoscopy or endotracheal intubation, which may be necessary to secure the airway.

On further questioning, you noted that Mrs Lim was recently started on aspirin following the diagnosis of type 2 diabetes mellitus. Chest radiography showed no abnormalities and her haemoptysis stopped once the aspirin was discontinued. She has been asymptomatic since then.

\section{TAKE HOME MESSAGES}

1. Haemoptysis is expectoration of blood originating from the tracheobronchial tree. 


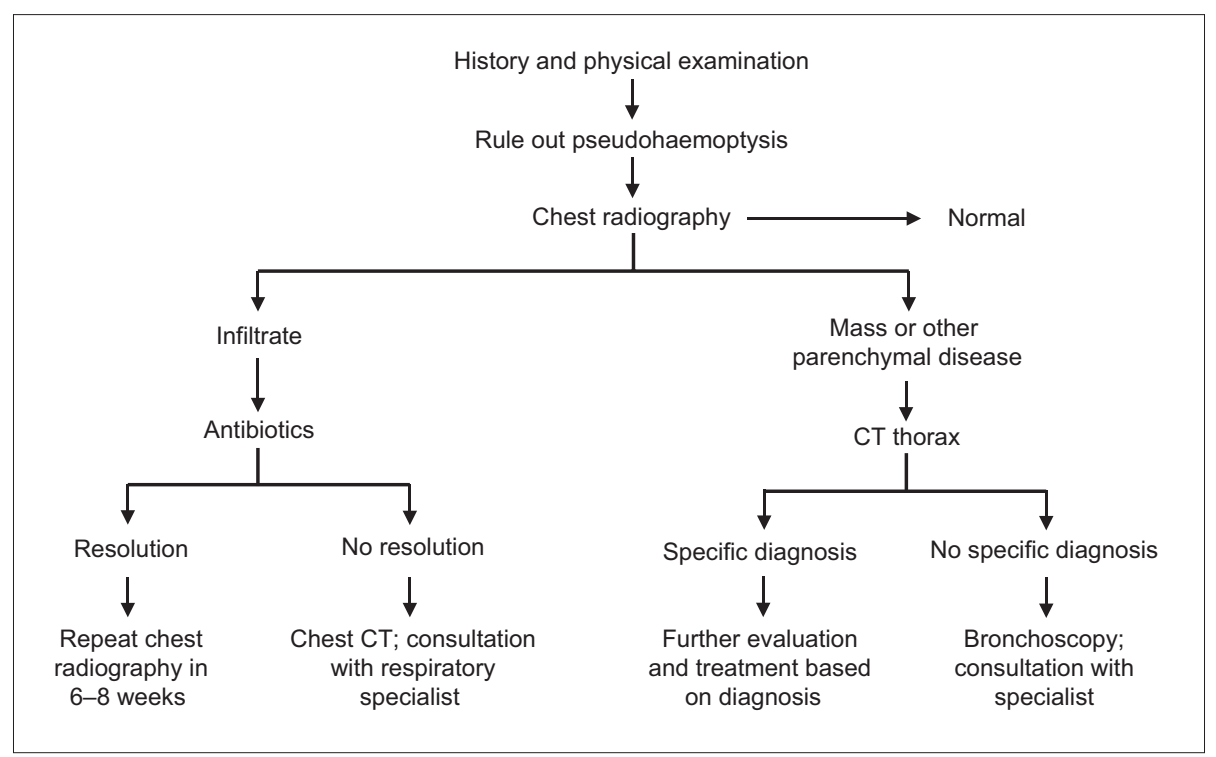

Fig. 1 Flowchart shows evaluation of non-massive haemoptysis. CT: computed tomography

2. It is important to differentiate pseudohaemoptsysis from true haemoptysis.

3. Causes of haemoptysis can be divided into five broad categories: infective, neoplastic, vascular, autoimmune and drug-related.

4. Chest radiography is recommended as a first-line investigation for all patients complaining of haemoptysis.

5. Massive haemoptysis is defined as loss of at least $200 \mathrm{~mL}$ of blood in 24 hours or $50 \mathrm{~mL}$ per episode. It warrants an inpatient or specialist referral.

ABSTRACT Haemoptysis is commonly seen in the healthcare setting. It can lead to life-threatening complications and therefore requires careful evaluation of the severity and status of the patient. Common causes of haemoptysis can be broadly grouped into five main categories: infective, neoplastic, vascular, autoimmune and drug-related. Detailed history-taking and careful physical examination are necessary to provide a diagnosis and assess the patient's haemodynamic status. Physicians must have a clear understanding of the criteria for further investigations and the need for a specialist or inpatient referral for management.

Keywords: aetiology, haemoptysis, primary care, pseudohaemoptysis

\section{REFERENCES}

1. Jeudy J, Khan AR, Mohammed TL, et al; Expert Panel on Thoracic Imaging. ACR Appropriateness Criteria hemoptysis. J Thorac Imaging 2010; 25:W67-9.

2. Weinberger SE, Lipson DA. Cough and hemoptysis. In: Fauci AS,
Braunwald E, Kasper DL, et al, eds. Harrison's Principles of Internal Medicine. 17th ed. New York: McGraw-Hill, 2008: 225-8.

3. Bidwell KL, Pachner RW. Hemoptysis: diagnosis and management. Am Fam Physician 2005; 72:1253-1260.

4. Larici AR, Franchi P, Occhipinti M, et al. Diagnosis and management of haemoptysis. Diagn Interv Radiol 2014; 20:299-309.

5. Abdulmalak C, Cottenet J, Beltramo G, et al. Haemoptysis in adults: a 5-year study using the French nationwide hospital administrative database. Eur Respir J 2015; 46:503-11.

6. Earwood JS, Thompson TD. Hemoptysis: evaluation and management. Am Fam Physician 2015; 91:243-9.

7. Cappell MS, Friedel D. Initial management of acute upper gastrointestinal bleeding: from initial evaluation up to gastrointestinal endoscopy. Med Clin North Am 2008; 491-509.

8. Ministry of Health, Singapore. World TB Day. In: Epidemiological News Bulletin 2016 Apr; 42. Available at: https://www.moh.gov.sg/content/dam/ moh_web/Publications/Epidemiological\%20News\%20Bulletin/ENB\%20 Quarterly_Apr\%202016_FINAL3.pdf. Accessed July 28, 2016.

9. Kumar P, Clark, M, eds. Kumar and Clark's Clinical Medicine, 8th ed. Edinburgh: Saunders, 2012: 791-866.

10. Shi CL, Zhou HX, Tang YJ, et al. [Risk factors of venous thromoboembolism recurrence and the predictive value of simplified pulmonary embolism severity index in medical inpatients]. Zhonghua Yi Xue Za Zhi 2016; 96:1112-5. Chinese.

11. Stein PD, Terrin ML, Hales CA, et al. Clinical, laboratory, roentgenographic, and electrocardiographic findings in patients with acute pulmonary embolism and no pre-existing cardiac or pulmonary disease. Chest 1991; 598-603.

12. Hoffman GS, Kerr GS, Leavitt RY, et al. Wegener granulomatosis: an analysis of 158 patients. Ann Intern Med 1992; 116:488-98.

13. Dammacco F, Battaglia S, Gesualdo L, Racanelli V, et al. Goodpasture's disease: a report of ten cases and a review of the literature. Autoimmun Rev 2013; 12:1101-8.

14. Von Feldt JM. Systemic lupus erythematosus. Recognizing its various presentations. Postgrad Med 1995; 97:79, 83, 86 passim.

15. Fartoukh M, Khoshnood B, Parrot A, et al. Early prediction of in-hospital mortality of patients with hemoptysis: an approach to defining severe hemoptysis. Respiration 2012; 83:106-14. 


\section{SINGAPORE MEDICAL COUNCIL CATEGORY 3B CME PROGRAMME} (Code SMJ 201608A)

1. Haemoptysis is defined as any blood expelled from the mouth by coughing.

2. Blood from massive nosebleeds that is coughed up through the mouth is the most common and harmless cause of haemoptysis.

3. True haemoptysis is only defined as blood loss from the tracheobronchial tree or pulmonary parenchyma.

4. Multiple episodes of haemoptysis with small amounts of blood that add up to an estimated volume loss of at least $200 \mathrm{~mL}$ or more in 24 hours is considered as massive haemoptysis.

5. Massive haemoptysis is rare and accounts for less than $1 \%$ of haemoptysis cases.

6. Massive haemoptysis has an associated mortality of above $75 \%$.

7. The five more common causes of haemoptysis can be grouped into infective, neoplastic, vascular, autoimmune and drug-related causes.

8. Pneumonia is not a common underlying cause for patients presenting with haemoptysis.

9. Tuberculosis is a common cause of haemoptysis, but not a common cause of massive haemoptysis.

10. Tuberculosis is a rare diagnosis with an incidence rate of approximately $1-2$ per 100,000 population in Singapore and should not be suspected in patients with haemoptysis.

11. Lung metastases that cause haemoptysis can come from other primary malignancies such as breast, kidney, gastrointestinal, ovarian and cervical cancers.

12. The presence of vasculitic rash, haematuria, joint pain or swelling may be suggestive of underlying autoimmune diseases that may contribute to haemoptysis.

13. Common drugs that may increase the risk of haemoptysis include anticoagulants and antiplatelet agents.

14. The presence of haemoptysis caused by anticoagulants and antiplatelet agents is a compelling reason to stop these medications, regardless of their indications.

15. A thorough clinical history-taking and physical examination in the primary care setting is important as it helps to narrow down the differential list and also quantify the amount of blood lost.

16. Chest radiography should be performed for every patient who presents with haemoptysis to screen for any focal or diffuse parenchymal involvement as well as pleural abnormalities.

17. When a patient with non-massive haemoptysis has a normal chest radiograph and no haemodynamic instability, no additional testing is required if the risk of massive bleed is low in the acute setting.

18. A patient presenting with haemoptysis who has a normal chest radiograph but is at increased risk of malignancy (i.e. 30 pack-years of smoking, age $\geq 40$ years) will still require computed tomography of the chest.

19. In patients presenting with massive haemoptysis with haemodynamic instability, the priorities of management should be the patient's airway, breathing and circulation.

20. While waiting for the ambulance, the patient should be placed in the lateral decubitus position with the affected lung in a dependent position to avoid pooling of blood in the unaffected lung.

\section{Doctor's particulars:}

Name in full

MCR number

Email address

\section{SUBMISSION INSTRUCTIONS:}

(1) Visit the SMJ website: http://www.smj.org.sg/current-issue and select the appropriate set of questions. (2) Provide your name, email address and MCR number. (3) Select your answers and click "Submit".

\section{RESULTS:}

(1) Answers will be published online in the SMJ October 2016 issue. (2) The MCR numbers of successful candidates will be posted online at the SMJ website by 3 October 2016. (3) Passing mark is $60 \%$. No mark will be deducted for incorrect answers. (4) The SMJ editorial office will submit the list of successful candidates to the Singapore Medical Council. (5) One CME point is awarded for successful candidates.

Deadline for submission: (August 2016 SMJ 3B CME programme): 12 noon, 26 September 2016. 